Eranga Perera, Henrik Petander, Aruna Seneviratne, Bandwidth fuelling for Network Mobility, in Proceedings of the Third IEEE and IFIP International Conference on Wireless and Optical Communications Networks (WOCN 2006), Bangalore, India, 1113 April 2006.

(C) 2006 IEEE

Reprinted with permission.

This material is posted here with permission of the IEEE. Such permission of the IEEE does not in any way imply IEEE endorsement of any of Helsinki University of Technology's products or services. Internal or personal use of this material is permitted. However, permission to reprint/republish this material for advertising or promotional purposes or for creating new collective works for resale or redistribution must be obtained from the IEEE by writing to pubs-permissions@ieee.org.

By choosing to view this document, you agree to all provisions of the copyright laws protecting it. 


\title{
Bandwidth fuelling for Network Mobility
}

\author{
Eranga Perera ${ }^{\Upsilon \pm}$ Student Member IEEE, Henrik Petander ${ }^{\uparrow+}$ Student Member IEEE, Aruna \\ Seneviratne ${ }^{\Upsilon}$ Member IEEE \\ $\Upsilon^{\prime}$ National ICT Australia, ATP, Bay 15, Locomotive Workshop Eveleigh, NSW 1430, Australia \\ ${ }^{ \pm}$Dept. EE \& Telecommunication University of New South Wales, Sydney Australia \\ ${ }^{\dagger}$ Department of Computer Science Helsinki University of Technology, Finland
}

\begin{abstract}
The deployment of mobile networks in automobiles, trains, ships, planes buses etc. would undoubtedly change the way we travel. More and more people would be inclined towards taking public transportation systems if they would get the opportunity to utilize their commuting time in a constructive manner. In order for on board Internet connectivity to be appealing for the public the cost and the performance of the service should be reasonable. However providing continuous high-performance service over wireless networks at a reasonable cost is still a challenge. Use of caching allows more efficient usage of the potentially scarce bandwidth of the wireless resources. In this paper we utilize the idea of caching and present a bandwidth fueling architecture for network mobility. We analyze the feasibility of the architecture by measuring the performance of our prototype implementation.

Index Terms - Mobile Cache Servers, NEMO Basic Support Protocol, Roadside WLAN Networks
\end{abstract}

\section{INTRODUCTION}

The number of embedded on board devices is growing at a staggering pace and at the same time most passengers carry mobile devices. A Mobile Router can be deployed to provide Internet connectivity for these devices via a wireless connection. Although in some environments it may be possible to use high bandwidth Wireless Local Area Network (WLAN) connections, in most cases it is necessary to use Wireless Wide Area Networks (WWAN) due to their larger coverage areas. Therefore, for the foreseeable future, mobile networking environments will consist of high bandwidth WLANs (Hotspots), overlaid with WWANs.

Use of a WWAN connection in Mobile Router provides continuous connectivity for on board devices. However, the high cost of bandwidth for WWAN links makes the use of bandwidth intensive services less attractive. In order to make these services more attractive for the users the cost of the communications should be minimized. Two techniques that have been used to minimize bandwidth usage and thereby the cost is pre-fetching and caching. Caching exploits the characteristic of locality, i.e. the fact that a number of people will want access to the same information, by storing data locally. Pre-fetching anticipates the demand for data and downloads the information in advance when the bandwidth costs are minimal.
Although use of caching and pre-fetching in mobile computing environments have been investigated in the past, to our knowledge, the feasibility of using it together with Mobile Routers have not been investigated.

In order for the Mobile Cache Server to provide a meaningful service, it is crucial for the caches to be updated frequently. However, updating of the Mobile Cache Server using WWAN connectivity would be slow, potentially costly and would compromise the real-time services, such as Voice over IP. Furthermore, performing frequent cache updates in a mobile network setting is a challenge in itself, due to mobility management overheads specific to the standardized network mobility protocol, NEMO [1].

In this paper we investigate the possibility of using Mobile Cache Servers in mobile computing environments which have WWAN overlaid over roadside hot spots. We incorporate similar ideas of Infostations [2] with an optimal routing mechanism that minimizes the overheads of the NEMO protocol [3]. This results in a complete bandwidth fuelling architecture for mobile networks. Then we show the viability of the proposed architecture through a proof of concept implementation of this architecture.

In the next section we will consider a few motivating scenarios for the proposed architecture. This would be followed by background and related work. In section IV the proposed architecture will be described. The implementation and the results will be given in section $\mathrm{V}$. We conclude the paper in Section VI.

\section{MOTIVATING SCENARIOS}

An on-board mobile network may contain both embedded devices, sensors and passenger devices. We will first consider how caching can benefit the flow of information between the embedded devices and the fixed network, and then briefly study the application of caching for customized services rendered for passengers.

On-board embedded devices and sensors are used to improve the performance, reliability and safety of vehicles. They can be also used for providing additional services, such as on-board advertisements and timetable services for bus or train stops. Many of these functions require or at least benefit 
from network connectivity. Some may have a need for interactive or real-time communications. These kinds of services cannot be improved using Mobile Cache Servers due to its periodic updates. However, in many cases, the information can be sent and received periodically. There are several examples of such services: An on-board device can monitor the condition of the vehicle and periodically update a service center on the current condition.

On-board video advertisements in commuter buses can be updated dynamically based on the target segments, which change according to the time of the day and at stops. Onboard Navigation systems can receive periodic updates about the traffic situations on its planned route. Use of an on-board Mobile Cache Server together with the Roadside Caches (localized caching in Roadside WLAN networks) will improve the performance of such applications.

Passengers can take advantage of the Mobile Cache Server in two distinct ways: 1) The Mobile Cache Server can provide the passengers with up to date services, such as news clips and tourist information. 2) Passengers can use the Mobile Cache Server to improve the performance of their own applications. Improving the performance of interactive or real-time network applications using a periodically updated Mobile Cache Server may be impractical. However, less interactive applications, such as file downloading and uploading can take advantage of the Cache Server. In the section IV we will describe how the Roadside architecture can be used to accelerate these operations.

\section{BACKGROUND AND RELATED WORK}

In this section we initially describe the mobility management protocol NEMO, which employs a special entity namely a Mobile Router to handle the mobility of an entire network. This will be followed by an introduction to a mobility optimization mechanism we utilize for efficient cache updates. Finally we present some related work on caching.

NEMO protocol is an extension to the MIPv6 host mobility protocol. In MIPv6 when a node moves in the Internet topology it sends an update of its current location in the form of a Binding Update to its Home Agent. The NEMO Basic Support protocol relies on the ability of the Mobile Router to convey the location updates to its Home Agent on behalf the entire network. The Mobile Router and its Home Agent uses bidirectional tunneling in order to preserve session continuity while the Mobile Router moves. The MIPv6 protocol has the desirable feature of optimal routing for the Mobile Network Nodes (MNNs), whereas with the NEMO Basic Support protocol there is no mechanism to enable route optimization. The non optimal routing via Home Agent hinders the performance of the system by increasing the Round Trip Time (RTT) between the communication end points and potentially introduces other side effects, such as packet loss, reordering and duplication, into the communications. The significance of these effects depends on the topological distance between the Home Agent and the end points of the communications. Fig. 1 depicts the non optimal routing path to and from a Correspondent Node to a Mobile Network Node that belongs to the Mobile Router's Home network. If the Mobile Network Node was for example a passenger's mobile device then all packets would traverse via two Home Agents. Since the Mobile Cache Server's home network would be the same as the Mobile Router's home network we do not consider routing via two Home Agents in this paper.

We employ a mechanism namely OptiNets RO proposed [3] and implemented [4] in our previous work to enable optimal routing between the Mobile Cache Servers and their Correspondent Nodes. This method relies on the Mobile Router to provide the foreign network prefix to the Mobile Network Nodes. Having a location specific address would enable the Mobile Cache Servers to perform direct routing with their Correspondent Nodes.

Use of caching to enhance the performance of wired and wireless networks is not a new concept. A number of studies have been conducted to reduce the Web traffic and overall network congestion by deploying various caching schemes in the wired Internet [5], [6]. Caching schemes such as [7], [8] are some of the techniques proposed for the wireless counterpart. However, to the best of our knowledge there has been no work on caching based techniques to improve the performance of network mobility such as the one we propose in this paper.

\section{The Proposed ARChitecture}

The architecture introduces two new entities in addition to Mobile Cache Servers namely Roadside Cache Servers and Roadside Routers. Roadside Routers would provide high bandwidth, low-latency WLAN access to the Roadside Cache Servers. The proposed bandwidth fuelling architecture with roadside networks is depicted in Fig. 2.

Normally Mobile Routers would be equipped only with a WWAN Egress interface. In order for it to connect directly to the Roadside network, it needs to be equipped also with a WLAN Egress interface.

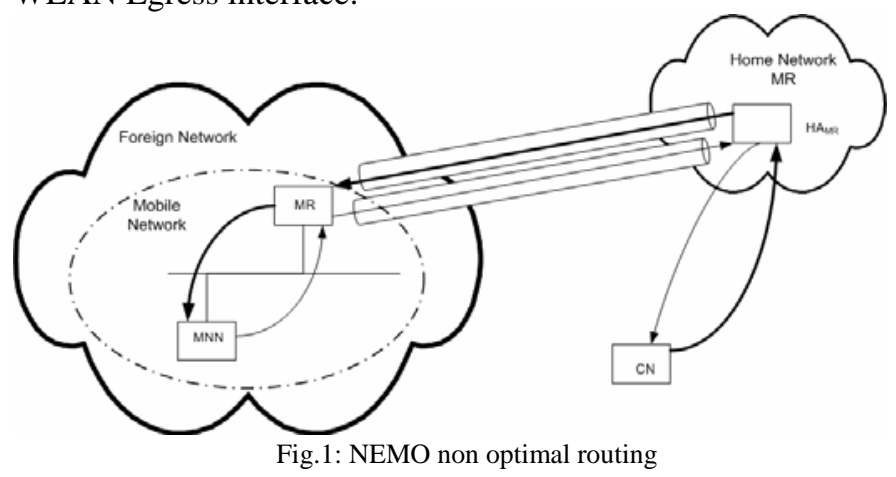




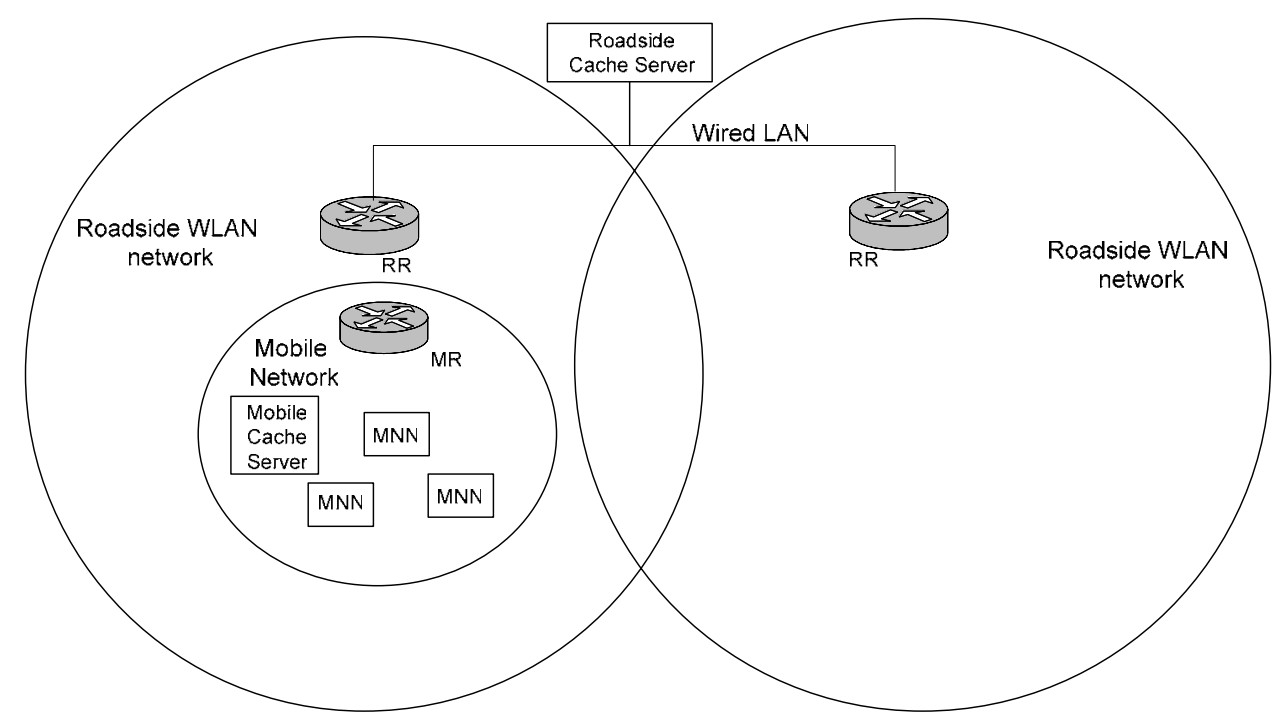

Fig 2: Bandwidth Fuelling Architecture for Mobile Networks

The fast WLAN connection together with the localized server allows the Mobile Cache Server to be updated during the time span that the Mobile Router is in the coverage area of the Roadside network. A Roadside Network could consist of one or more Roadside Routers connected to a Roadside Cache Server. We will give the technical aspects of the architecture by first considering access to a Roadside Cache Server via a single Roadside Router while the mobile network is static, i.e. the vehicle has stopped. This will be followed by the case of using a cluster of Roadside Routers for access while the vehicle with the mobile network is moving.

We assume that the Mobile Routers are preconfigured with the identities of Roadside networks controlled by their Roadside access providers. Therefore the Mobile Router can detect the availability of Roadside Routers belonging to these networks and connect. It can then acquire a block of addresses from the Roadside Network and delegate an address to the Mobile Cache Server as proposed in the OptiNets Route optimization mechanism. The Mobile Cache Server then uses the location specific address from the roadside network to communicate directly with the Roadside Cache Server. This is in contrast to the indirect routing normally used with NEMO where packets to and from the mobile network are routed via the home network of the Mobile Router. Fig. 3 depicts the signaling diagram for the route optimization procedure using the OptiNets mechanism. The optimized routing path reduces the Round Trip Time, thus improving TCP throughput. Especially in a wireless setting with varying Round Trip Times, the RTT has a large effect on TCP [9] .

In the case where the mobile network is within the coverage area of a Roadside Network consisting of multiple Roadside Routers the Mobile Cache Server needs to maintain the connectivity to the Roadside Cache Server for the duration of the cache update. Although this could be achieved with the NEMO tunneling via the Mobile Router and its Home Agent as shown before, this would potentially mitigate the benefits of using a Roadside Cache Server. Therefore as in the static case we use the OptiNets RO mechanism to enable the Mobile Cache Server to obtain a location specific address via the Mobile Router. In order for the Mobile Cache Server to benefit from the location specific address and perform direct routing when the network is moving the Mobile Cache Server needs to be MIPv6 capable. Having the MIPv6 capability would enable the Mobile Cache Server to use the location specific address and communicate directly with the Roadside Cache Server.

Upon receiving a request for data, the Mobile Router would first attempt to retrieve the information from the Mobile Cache Server. If the information is not available on the Mobile Cache Server, the Mobile Router would use its WWAN connection and serve the device. If the Mobile Router is unable to handle the request at the given time, it will inform the mobile device of the delay in serving the request. The architecture can be optimized to handle such situations. For example, the Mobile Router could request the Mobile Cache Server to start pre-fetching the information as soon as possible. The idea of pre-fetching via the Mobile Cache Server could be extended to enable customized services such as online audio or video clips.

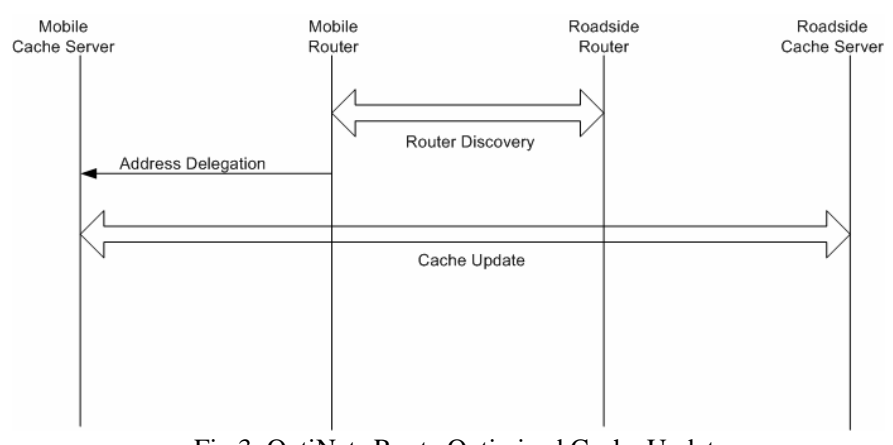

Fig 3: OptiNets Route Optimized Cache Updates 
We propose the idea of using context information in order to enable pre-fetching of data to a Roadside Cache Server before the Mobile Network enters the road side network coverage area. This context information can be based on a combination of geographical context and vehicle route information. Mobile Router uses the context information and identifies the network it is about to enter and looks up the address of the corresponding Roadside Cache Server. It then informs the Mobile Cache Server of the address of the Roadside Cache server. Using this address, Mobile Cache Server sends a request for pre-fetching to the Roadside Cache Server using NEMO tunneling. Successful pre fetching of data to the Roadside Cache Servers would ensure that the customized data are ready to be downloaded efficiently using the low cost WLAN connection while the mobile network is in a coverage area of the Roadside network.

Timely fetching of customized data to the Roadside Cache Server depends on the ability to predict mobility correctly. However, even without mobility prediction the Roadside Cache Server would be able to provide the Mobile Cache Server with fresh non-customized data, such as local news, tourist information and traffic information.

\section{IMPLEMENTATION AND RESULTS}

We have implemented the Bandwidth fuelling architecture for network mobility using our Linux-based NEMO implementation as a basis. We utilize DHCP for prefix delegation between Mobile Router and Roadside Router, so that the Mobile Router receives a prefix from the Roadside Router upon attaching to it. As described in the previous section, Mobile Cache Server configures itself with an address from this network prefix and uses this address to communicate with the Roadside Cache Server.

Mobile Cache Server uses Mobile IPv6 to maintain its connection to the Roadside Cache Server, when it moves through the Roadside Network, switching between multiple Roadside Routers. To minimize the disruption caused by the handoffs, we also extended the NEMO protocol to better support frequent handoffs. We have analyzed the effects of the handoffs on TCP performance in [4]. We measured the effectiveness of the road side caching architecture using our mobile network test bed [10]. Our test network consists of WLAN access networks connected to Roadside Routers, a server node and the Home Agent of the Mobile Router.

Initially we show the benefit of using a Roadside Cache Server as opposed to using a remote server for cache updates. We do this by comparing the relative TCP performance of a static (i.e. when the mobile network has stopped) cache update when using a Roadside Cache Server to using a remote cache server. We simulate the remoteness of the server by using a Wide Area Network (WAN) emulator, NISTNet and measure the effects of varying RTT on TCP performance using the iperf performance analysis tool. We used the default TCP settings in the Linux 2.4.26 kernel for all the measurements.
We used NISTNet to modify the network latency between the Roadside Router and the remote server. The network latency between Mobile Cache Server and Roadside Router was $2.5 \mathrm{~ms}$ in all the measurements, which can be considered as negligible. Therefore we consider the topological distance between the Roadside Router and the remote server to be the same as the distance between the Mobile Cache Server and the remote server.

Fig. 4 illustrates the TCP throughput as a function of topological distance between the Mobile Cache Server and a remote server. It clearly depicts that as the distance increases the TCP performance degrades significantly when compared to using a localized server, thus confirming the viability of using a Roadside Cache Server. The measurements take into account only the effects of RTT on TCP, but remote connections may suffer from further degradation due to packet duplication, reordering and packet loss. Furthermore use of a Roadside Cache Server allows a Mobile Cache Server to retrieve all information in a compressed form, instead of gathering information piecewise, e.g. retrieving web pages by following hyperlinks.

We analyze the effects of mobility on the cache updating process as described in Section IV. The Roadside network would consist of a one-dimensional, linear array of WLAN cells as depicted in Fig. 5. A Roadside Router equipped with a WLAN antenna would act as the access point for each cell. For mobile updating of the Mobile Cache Server, Mobile Router needs to manage the mobility to maintain the connection between the Mobile Cache Server and Roadside Cache Server. We utilize dual interfaces in Mobile Router and overlapping WLAN cells to ensure seamless connectivity as described in [11]. We combine the seamless handoffs with OptiNets routing to maximize the performance of cache updates. We will first consider a case of very frequent mobility in which a Mobile Router performs a handover to a new Roadside Router every 5s. This handoff frequency corresponds to a vehicle moving at $150 \mathrm{~km} / \mathrm{h}$ in a Roadside network with a cell size of $250 \mathrm{~m}$.

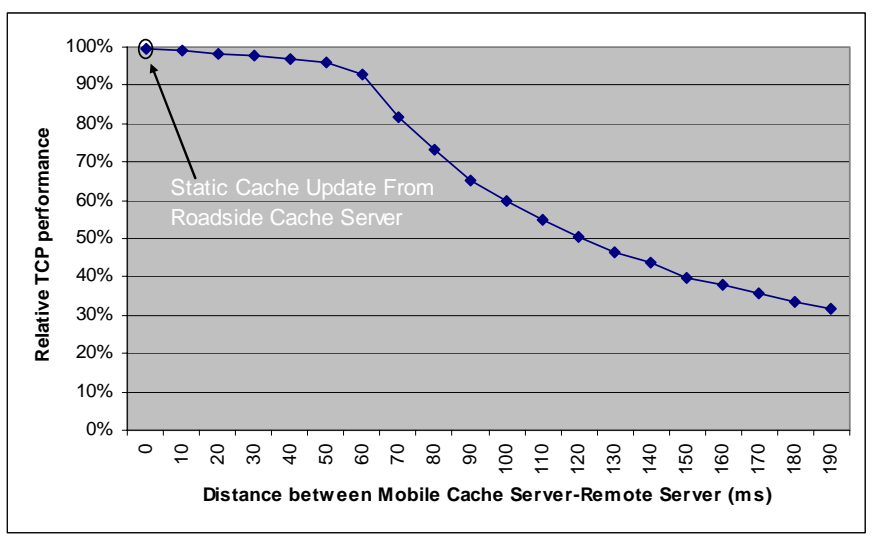

Figure 4: Relative TCP performance of a static cache update as a function of distance to a remote server, with $100 \%$ depicting the performance of a Roadside Cache Server 


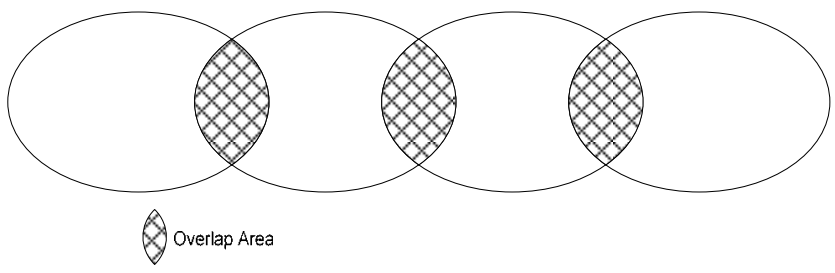

Figure 5: One-Dimensional linear topology model

We analyze the effects of the mobility on the cache updates by comparing the performance of a mobile update with an update which is performed when the mobile network is static. We use normal NEMO routing and a combination of Optinets scheme and seamless handoffs for the comparison. We perform the comparison using different network topologies that is by varying the distance between home network and current location of Mobile Router.

We measure the performance of the TCP connection between Mobile Cache Server and Roadside Cache Server for 10s. In the measurements Mobile Router is first attached to one Roadside Router and Mobile Cache Server starts to synchronize its cache with the Roadside Cache Server. After $5 \mathrm{~s}$ the Mobile Router performs a handoff to a new Roadside Router. Fig. 6 depicts the effects of mobility on the cache update performance with normal NEMO routing and OptiNets routing. The results are given as a relative TCP performance to the static cache update with a Roadside Cache Server. It is evident from the results that the relative TCP performance is very close to the case of static cache updates with OptiNets cache updates as opposed to normal NEMO cache updates.

The measurement settings for the mobile case emulate a mobile network moving at a high speed between small cells. Use of directional antennas would provide more efficient coverage of a road or railroad, and thus it is likely that the cell size would be in estimated range of one to several kilometers [12]. This would result in less frequent handoffs, and thus the handoff frequency of one handoff in every 5 seconds which we have used for our measurements can be considered as a worst case scenario. From the obtained results (depicted in figure 7) it is evident that even in the worst case scenario of a handoff frequency of 1 in every 5 seconds the relative TCP performance is very close to the static case.

Our prototype implementation of the proposed architecture confirmed the feasibility of this bandwidth fuelling mechanism for network mobility. Further the results presented in this section shows that a Roadside network architecture together with the network mobility optimizations provides a means for efficient cache updates for a Mobile Cache Server.

\section{CONCLUSION}

In this paper we proposed a bandwidth fuelling architecture for mobile networks. We considered the issue of reducing the usage of high cost WWAN links in a network mobility setting. We proposed the utilization of Mobile Cache Servers together with low cost WLAN roadside wireless networks in order

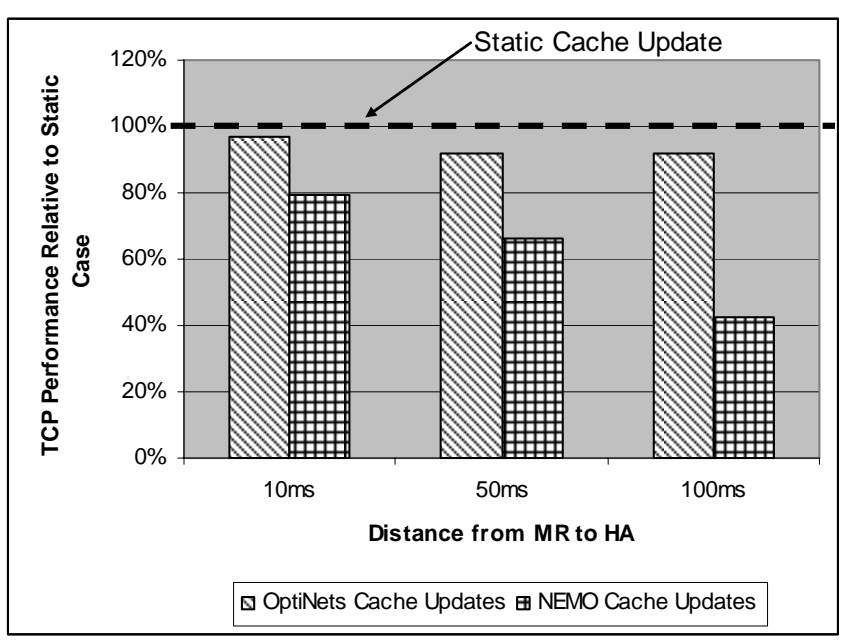

Figure 6: TCP performance of mobile cache updates relative to static updates as a function of distance from MR to Home Agent

enable Mobile Routers to provide a low cost service to the devices within a mobile network. As future work we will integrate the idea of pre fetching using mobility predictions to our implementation.

\section{REFERENCES}

[1] Devarapalli V., Wakikawa R., Petrescu A., Thubert P. "NEtwork MObility (NEMO) Basic Support Protocol”, Internet RFC, RFC 3963, January 2005.

[2] Goodman D., Borras J.,Mandayam N.B., Yates R., "INFOSTATIONS : A New System Model for Data and Messaging Services", Proceedings of IEEE VTC'97, vol. 2, pp. 969-973,May 1997

[3] Perera E., Seneviratne A., Sivaraman V., "OptiNets: An architecture to enable optimal routing in network mobility", International Workshop on Wireless Ad-Hoc Networks, May 2004.

[4] Petander H., Perera E., Seneviratne A., "Measuring and improving performance of network mobility management in IPv6 networks ", NICTA Technical Report, http://nicta.com.au/director/research/ publications/technical reports/2005.cfm

[5] L. Fan, P. Cao, J. Almeida, and A. Z. Broder, "Summary Cache: A Scalable Wide-Area Web Cache Sharing Protocol," in Proc. ACM, SIGCOMM, 1998, pp. 254-265.

[6] K. Wu, P. S. Yu, and J. L. Wolf, "Segment-Based Proxy Caching of Multimedia Streams," in Proc. World Wide Web (WWW 10), May 2001, pp. 36-44.

[7] Y. Hu and D. B. Johnson, "Caching Strategies in On-Demand Routing Protocols for Wireless Ad Hoc Networks," in Proc. ACM MOBICOM, 2000, pp. 231-242.

[8] Valera A., Winston K., Rao S., "Cooperative Packet Caching and Shortest Multipath Routing in Mobile Ad hoc Networks" in Proc. IEEE Infocom, 2003.

[9] Abouzeid A., Roy S., Azizoglu M., "Comprehensive Performance Analysis of a TCP Session Over a Wireless Fading Link With Queueing", IEEE Transactions on Wireless Communications, Vol. 2, No. 2, March 2003.

[10] Lan K., Perera E., Petander H., Dwertmann C., Libman L., Hassan M., "MOBNET: The Design and Implementation of a Network Mobility Testbed", IEEE LANMAN, Sep. 2005.

[11] Petander H., Perera E., Seneviratne A., "Multiple Interface Handoffs: A Practical Method for Access Technology Independent Make-BeforeBreak Handoffs", NICTA Technical Report, http://nicta.com.au/ director/research/publications/technical_reports/2005.cfm

[12] U.S. Robotics, Technical Report, "Wireless Networking : Using Antennas to Extend and Enhance Wireless Connections", http://shop.renaissance.co.nz/catalogue/hosted/usr/technote-antennae.pdf 